\title{
MÉTODOS DE DETERMINAÇÃO DE DANOS EM FLORESTAS PLANTADAS
}

\author{
Alessandro Albino Fontes - Dr. em Ciência Florestal - Universidade Federal de Viçosa, \\ aafontes@vicosa.ufv.br \\ Márcio Lopes da Silva - Professor do Departamento de Engenharia Florestal - \\ Universidade Federal de Viçosa, marlosil@ufv.br \\ Sidney Araujo Cordeiro - Doutorando em Ciência florestal - Universidade Federal de Viçosa, \\ sidney.cordeiro@ufv.br
}

\section{RESUMO}

Objetivou-se revisar e comparar alguns métodos para a avaliação de danos a áreas florestais como: valor do custo, valor produtivo da terra, valor de mercado, método de McLean e o método alternativo de Cárcamo Romero. Para isto, considerou-se uma área reflorestada com Eucalyptus grandis, espaçamento $3 \times 2 \mathrm{~m}$, incremento médio anual de $30 \mathrm{~m}^{3} / \mathrm{ha} / \mathrm{ano}$, com horizonte de planejamento de um único corte (aos 7 anos). Após simulação, concluiu-se que os métodos estudados, a exceção do valor de mercado (VM), proporcionaram estimativas consistentes para o dano a áreas florestais e pouco diferindo entre si. Assim, a decisão de qual método utilizar dependerá dos objetivos do manejador. Palavras-chave: danos florestais; avaliação florestal; reflorestamento de eucalipto.

\section{DAMAGE EVALUATION METHODS IN PLANTED FORESTS}

\section{ABSTRACT}

The objective was to review and compare some methods of evaluating damages to forest areas such as: cost value, land productive value, market value, McLean method and the alternative Cárcamo Romero method. For this purpose an area planted with Eucalyptus grandis, with a spacing of $3 \times 2 \mathrm{~m}$, with planning horizon of a single harvest ( 7 years. After simulation, it was concluded that, except for the market value (VM), the studied methods provide consistent estimations for the damages to forest areas and with little difference among them. Therefore, the decision about which method to use will depend on the objectives of the manager.

Key-words: forest damages; forest evaluation; eucalypt reforestation.

\section{INTRODUÇÃO}

O plantio de florestas vem crescendo a cada ano a fim de suprir o consumo de madeira para os mais diferentes fins industriais no Brasil. Aproximadamente $66,1 \%$ do consumo refere-se a madeira de eucalipto $\left(103,3\right.$ milhões $\left.\mathrm{m}^{3}\right)$ e $33,9 \%$ de pinus $\left(52,9\right.$ milhões $\left.\mathrm{m}^{3}\right)$. O principal segmento consumidor é a indústria de celulose e papel (29,9\%), seguida pela siderurgia $(22,1 \%)$ e pela indústria de madeira serrada $(19,1 \%)$. A participação das indústrias de compensado e de painéis reconstituídos representa no total cerca de $10 \%$ (Abraf, 2007).

No Brasil, a ocorrência de pragas, doenças e incêndios tem-se verificado não só nas áreas reflorestadas, mas também em áreas de florestas nativas. Embora não exista uma estatística oficial, anualmente, uma porção considerável de florestas são danificadas pelos mais diversos fatores, causando prejuízos econômicos, ambientais e sociais, sendo, portanto, de grande importância o conhecimento da 
magnitude destes.

Os danos aos recursos florestais podem ter inúmeras causas e podem afetar, além do produto madeira, outros recursos vinculados à floresta, tais como: recreação, vida silvestre, fauna, etc. Podem afetar as condições de vida de uma região, pelos efeitos no microclima, em fontes de água, no solo, na proteção e apoio à outras culturas e até mesmo o número de empregos (Cárcamo Romero, 1985).

Dentre os principais agentes causadores de danos às florestas estão: incêndios, doenças, pragas (insetos), pastoreio intensivo, vegetação indesejável, fauna e fatores climáticos externos, tais como: temperatura, chuva, vento, geada, raios e neves, e ainda os danos por poluição causada por resíduos lançados pelas indústrias no solo, ar e água (Ciancilli, 1981).

O valor do dano é definido como a diferença entre o valor da propriedade ou do povoamento antes da ocorrência do dano e o valor da propriedade ou povoamento após a ocorrência do dano. Este valor, seja ele acidental, natural ou deliberadamente provocado, pode ser estimado em termos monetários apenas para alguns produtos da floresta (Mclean, 1970).

As avaliações econômicas nas áreas danificadas são de grande importância no sentido de darem uma idéia correta dos prejuízos financeiros causados pelos diversos tipos de dano às florestas brasileiras. Permite que empresas públicas e privadas determinem, de forma objetiva, orçamentos para prevenção e controle de incêndios, propicia estabelecer níveis de risco em investimentos futuros e fornece guias operacionais ao processo de planejamento.

Nesse sentido, este estudo teve como objetivo revisar e comparar alguns métodos para a avaliação de danos a áreas florestais.

\section{MATERIAL E MÉTODOS}

Para simulação dos métodos de avaliação de danos a áreas florestais mencionados, considerou-se uma área reflorestada com Eucalyptus grandis W. Hill ex Maiden, espaçamento 3x2m, incremento médio anual de $30 \mathrm{~m}^{3} / \mathrm{ha} /$ ano e colheita os 7 anos.

A produção, preço e custos das atividades do reflorestamento foram levantados através de pesquisas utilizando-se dados médios obtidos de empresas florestais, na Embrapa Florestas, Cedagro, em setembro de 2008. O preço da madeira destinada para geração de energia, após os 4 anos de idade, foi US\$16,00/ $\mathrm{m}^{3}$. Os custos são apresentados no Quadro 1.

Neste estudo considerou-se uma taxa de juros de $6 \%$ a.a., que é a taxa de juros de empréstimo de capital inicialmente adotado pelo programa Propflora do Banco do Brasil e demais bancos credenciados pelo BNDES, para investimento e produção de florestas.

QUADRO 1. Custos do Projeto de reflorestamento com eucalipto.

TABLE 1. Costs to reforestation eucalypt project production.

\begin{tabular}{|c|c|c|}
\hline Atividade & $\begin{array}{c}\text { Ano de } \\
\text { ocorrência }\end{array}$ & $\begin{array}{c}\text { Custos } \\
\text { (US\$/ha) }\end{array}$ \\
\hline Implantação & 1 & 780,00 \\
\hline Manutenção & 2 a 6 & 80,00 \\
\hline Colheita & 7 & 840,00 \\
\hline Custo anual da terra & 1 a 7 & 130,00 \\
\hline
\end{tabular}

Fonte: Organizado pelos autores a partir de dados de Embrapa Florestas (2008) e Cedagro (2008). US\$1.00 igual a $\mathrm{R} \$ 1,90$.

Considerou-se o valor da terra como sendo de US\$ $1.000,00 /$ ha e o custo anual da terra (CAT) estimado como sendo os juros (i) sobre o valor da terra (VT), conforme a seguinte fórmula:

$$
\mathrm{CAT}=\mathrm{VT}^{*} \mathrm{i}
$$

\section{Métodos de avaliação de danos}

Muitos autores estudaram o problema da estimativa ou avaliação econômica dos danos 
causados por incêndios em áreas florestais. Alguns consideraram como medida do dano o valor da madeira danificada, enquanto outros incluíram, além do valor da madeira, os danos causados a outros recursos da propriedade ou relacionados à madeira (Sparhawk, 1925; Craig, 1945; Mactavish, 1965; Marty, 1965; McLean, 1970; Crosby, 1977; Gorte e Ross, 1979; Wagner, 1979; citados por Cárcamo Romero, 1985). A maioria destes métodos vem sendo utilizados em áreas de florestas nativas ou de floresta manejadas com poucas espécies e longas idades de rotação, principalmente em países como o Canadá e os Estados Unidos.

Os métodos para avaliação de danos a áreas florestais utilizados neste estudo foram: o valor do custo; valor produtivo da terra e o valor de mercado, propostos por Davis (1965); o método proposto por Mclean (1970) e o método alternativo sugerido por Cárcamo Romero (1985).

\section{Método do valor do custo (VC)}

O valor do custo, proposto por Davis (1965), é baseado no valor atualizado, até o memento da ocorrência do dano, de todos os custos históricos de implantação e de manutenção da floresta, conforme a expressão:

$$
V C_{t}=C I(1+i)^{Y}+\frac{R\left[(1+i)^{\prime}-1\right\rfloor}{i}
$$

em que:

$\mathrm{VC}_{\mathrm{t}}=$ valor do custo no momento de ocorrência do dano, t, em R $\$ /$ ha;

$\mathrm{CI}=$ custo de implantação, em $\mathrm{R} \$ /$ ha;

$\mathrm{R}=$ custos anuais, em $\mathrm{R} \$ / \mathrm{ha}$;

$\mathrm{i}=$ taxa de juros, em $\%$ ao ano;

$\mathrm{t}=$ idade do povoamento no momento de ocorrência do dano, em anos.

\section{Método do valor produtivo da terra (VPT)}

O valor produtivo da terra, também proposto por Davis (1965), é baseado no valor presente, no momento da ocorrência do dano, da diferença entre as receitas e os custos futuros estimados, podendo ser obtido através da equação:

$$
V P T_{t}=\operatorname{Re}_{n}(1+i)^{-(n-t)}-\frac{R\left[1-(1+i)^{-(n-t)}\right]}{i}-C C_{n}(1+i)^{-(n-t)}
$$

em que:

$\mathrm{VPT}_{\mathrm{t}}=$ valor produtivo da terra no momento de ocorrência do dano, t, em $\mathrm{R} \$ /$ ha;

$\mathrm{Re}_{\mathrm{n}}=$ receita na idade de rotação, $\mathrm{n}$, em $\mathrm{R} \$ / \mathrm{ha}$; $\mathrm{CC}_{\mathrm{n}}=$ custo de colheita na idade de rotação, $\mathrm{n}$, em R\$/ha;

$\mathrm{n}$ = idade de rotação do povoamento, em anos;

$\mathrm{R}, \mathrm{i}$ e $\mathrm{t}=$ definidos anteriormente.

\section{Método do valor de mercado (VM)}

O preço de mercado é um guia apropriado para estimar o valor da propriedade florestal e apresenta a vantagem de incorporar todos os fatores que afetam o valor do dinheiro (Davis, 1965).

Neste estudo, considerou-se o valor de mercado como sendo o valor do povoamento florestal no momento de ocorrência do dano, estimado multiplicando-se o estoque (volume) de madeira pelo preço de mercado.

\section{Método McLean}

Este método considera que o valor do dano a áreas florestais deve ser estimado pela madeira danificada, mas pode ser aplicado a florestas homogêneas e de mesma idade, ainda que os plantios não tenham atingido a idade comercial (Mclean, 1970).

Segundo Mclean (1970), o problema central de toda avaliação econômica de danos é determinar o valor do plantio antes e depois da ocorrência do dano, sendo o valor do dano definido como a diferença entre o valor de custo e o de expectativa da terra, ou seja,

em que:

$$
\mathrm{D}_{1}=\mathrm{V}_{\mathrm{t}}-\mathrm{S}_{\mathrm{e}}
$$

$\mathrm{D}_{1}=$ valor do dano, em $\mathrm{R} \$ /$ ha; 
$\mathrm{V}_{\mathrm{t}}=$ valor de custo, em $\mathrm{R} \$ / \mathrm{ha}$;

$\mathrm{S}_{\mathrm{e}}=$ valor de expectativa da terra, em $\mathrm{R} \$ /$ ha.

\section{a) Valor de expectativa da terra ( $\mathrm{Se}$ )}

As terras destinadas à produção florestal tem seus valores de acordo com as rendas ou benefícios que proporcionam a seus proprietários. $\mathrm{O}$ valor de expectativa da terra é o valor descontado da receita líquida a ser recebida no final de cada rotação, considerando um horizonte infinito. A receita líquida representa a diferença, na idade de rotação ou de corte, entre as receitas totais e os custos totais. É dada pela seguinte fórmula:

$$
S e=\frac{\operatorname{Re}_{n}-C I(1+i)^{n}-\frac{R\left[(1+i)^{n}-1\right]}{i}-C C_{n}}{(1+i)^{n}-1}
$$

em que:

$\mathrm{Re}_{\mathrm{n}}=$ receitas acumuladas até a idade de rotação, n, em R $\$$ ha;

$\mathrm{S}_{\mathrm{e}}, \mathrm{CI}, \mathrm{R}, \mathrm{CC}_{\mathrm{n}}, \mathrm{i}$ e $\mathrm{n}=$ definidos anteriormente.

\section{b) Valor de custo (Vt)}

Este conceito considera o valor das receitas e o valor conjunto da terra e dos custos de implantação e manutenção até a idade de avaliação. $O$ valor de custo é dado pela diferença entre o valor de todos os custos e o valor das receitas, valores estes acumulados até a idade de avaliação, "t".

$\mathrm{O}$ valor de custo inclui tanto o valor do material lenhoso como o do investimento em terra, sendo que este último é representado pelo valor de expectativa da terra (Se). A fórmula de cálculo é:

$$
V_{t}=(S e+C I)(1+i)^{\prime}+\frac{R\left[(1+i)^{\prime}-1\right]_{+}}{i}+C C_{t}-\mathrm{Re}_{t}
$$

em que:

$\mathrm{CC}_{\mathrm{t}}=$ custo de colheita na idade de avaliação, t, em $\mathrm{R} \$ /$ ha;

$\mathrm{Re}_{\mathrm{t}}=$ receitas acumuladas até a idade de avaliação, t, em R $\$ /$ ha;
$\mathrm{V}_{\mathrm{t}}$, Se, CI, R, i e $\mathrm{t}=$ definidos anteriormente.

\section{Método alternativo de Cárcamo Romero}

Cárcamo Romero (1985), propôs um método alternativo, com base no método de McLean, que permite às empresas florestais determinar o valor dos danos em povoamentos com base num valor da terra diferente de seu valor de expectativa, devido às distorções de mercado. Sua fórmula é expressa por:

$$
\mathrm{D}_{2}=\mathrm{VSD}-\mathrm{VCD}
$$

em que:

$\mathrm{D}_{2}=$ valor do dano, em $\mathrm{R} \$ / \mathrm{ha}$;

VSD $=$ valor do povoamento antes de ocorrer o dano, em $\mathrm{R} \$ / \mathrm{ha}$;

$\mathrm{VCD}=$ valor do povoamento após ocorrer o dano, em R\$/ha.

O valor do povoamento antes de ocorrer o dano (VSD), considerando um horizonte de planejamento de um ciclo de corte, é dado pela diferença entre o valor das receitas e o valor dos custos, ambos acumulados até a idade de rotação e descontados, a seguir, para a idade de avaliação. A fórmula é:

$$
V S D=\frac{\operatorname{Re}_{n}-C I(1+i)^{n}-\frac{R\left[(1+i)^{n}-1\right]}{i}-I T\left[(1+i)^{n}-1\right]-C C_{n}}{(1+i)^{n-t}}
$$

em que:

$\mathrm{IT}=$ investimento em terra, em $\mathrm{R} \$ / \mathrm{ha}$;

VSD, $\mathrm{Re}_{\mathrm{n}}, \mathrm{CI}, \mathrm{R}, \mathrm{CC}_{\mathrm{n}}, \mathrm{i}, \mathrm{n}$ e t $=$ definidos anteriormente.

O valor do povoamento após a ocorrência do dano (VCD), para um horizonte de planejamento de um ciclo de corte, é dado pela seguinte fórmula:

$$
V C D=\mathrm{Re}_{t}-C I(1+i)^{y}-\frac{R\left[(1+i)^{y}-1\right]}{i}-I T\left[(1+i)^{y}-1\right]-C C_{t}
$$

em que:

VCD, $\mathrm{Re}_{\mathrm{t}}, \mathrm{CI}, \mathrm{R}, \mathrm{IT}, \mathrm{CC}_{\mathrm{t}}$, i e $\mathrm{t}=$ definidos 
anteriormente.

O dano pode ocorrer antes de se obter alguma receita. Para o caso do eucalipto, normalmente o ciclo é de três rotações, o VCD pode ser positivo ou negativo.

\section{RESULTADOS E DISCUSSÃO}

Após a simulação dos métodos de avaliação de danos a áreas florestais abordados, para o cenário considerado, seguem-se as estimativas do parâmetros (Quadro 2).

Deve-se esclarecer que aos 7 anos de idade do povoamento houve ocorrência do dano antes da realização da colheita. Caso contrário, o valor do dano para este período seria zero.

QUADRO 2. Estimativas dos parâmetros dos métodos de avaliação de danos a áreas florestais.

TABLE 2. Estimation of the parameters of damage evaluation methods to forest areas.

\begin{tabular}{|c|c|c|c|c|c|c|c|c|c|}
\hline Ano & $\begin{array}{c}\mathrm{VC} \\
\text { (US\$/ha) }\end{array}$ & $\begin{array}{c}\text { VPT } \\
\text { (USS/ha) }\end{array}$ & $\begin{array}{c}\text { VM } \\
\text { (US\$/ha) }\end{array}$ & $\begin{array}{c}\text { Vt } \\
\text { (USS/ha) }\end{array}$ & $\begin{array}{c}\mathrm{Se} \\
\text { (US\$/ha) }\end{array}$ & $\begin{array}{c}\text { D1 } \\
\text { (US\$) }\end{array}$ & $\begin{array}{c}\text { VSD } \\
\text { (USS/ha) }\end{array}$ & $\begin{array}{c}\text { VCD } \\
\text { (US\$ } / \text { ha) }\end{array}$ & $\begin{array}{c}\text { D2 } \\
\text { (USS/ha) }\end{array}$ \\
\hline 1 & 966,8 & $1.088,08$ & -.. & $2.328,88$ & $1.341,58$ & 987,29 & 121,28 & $-966,8$ & $1.088,08$ \\
\hline 2 & $1.164,81$ & $1.293,36$ & 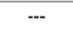 & $2.548,61$ & $1.341,58$ & $1.207,03$ & 128,55 & $-\overline{1}$ & $1.293,36$ \\
\hline 3 & $1.374,70$ & $1.510,96$ & -- & $2.781,53$ & $1.341,58$ & $1.439,94$ & 136,26 & $\begin{array}{c}- \\
1.374,70\end{array}$ & $1.510,9$ \\
\hline 4 & $1.597,18$ & $1.741,62$ & $1.920,00$ & $3.028,42$ & $1.341,58$ & $1.686,84$ & 144,44 & $\begin{array}{c}- \\
1.597,18\end{array}$ & \begin{tabular}{|l|l|}
$1.741,62$ \\
\end{tabular} \\
\hline 5 & $1.833,01$ & $1.986,12$ & $2.400,00$ & $3.290,12$ & $1.341,58$ & $1.948,54$ & 153,11 & $1.833,01$ & $1.986,12$ \\
\hline 6 & $2.082,99$ & $2.245,28$ & $2.880,00$ & $3.567,53$ & $1.341,58$ & $2.225,95$ & 162,29 & $2.082,99$ & $2.245,28$ \\
\hline 7 & $2.347,97$ & $2.520,00$ & $3.360,00$ & $3.861,58$ & $1.341,58$ & $2.520,00$ & 172,03 & $\begin{array}{c}-\dot{-} \\
2.347,97\end{array}$ & $2.520,0$ \\
\hline
\end{tabular}

Onde: valor do custo (VC), valor produtivo da terra (VPT), valor de mercado (VM), valor de custo (VT), valor de expectativa da terra (Se), Valor do dano pelo método de McLean (D1), valor do povoamento antes da ocorrência do dano (VSD), valor do povoamento após a ocorrência do dano (VCD) e valor do dano pelo método de Cárcamo Romero (D2).

\section{Método do valor do custo (VC)}

Este método é relativamente simples e permite uma estimativa satisfatória para o dano em povoamentos florestais imaturos, onde ainda não ocorreu colheita e, consequentemente, receitas. Conforme definido anteriormente, ele baseia-se no valor atualizado, até o memento da ocorrência do dano, de todos os custos de implantação e de manutenção da floresta não contemplando possíveis receitas.

\section{Método do valor produtivo da terra (VPT)}

Para o cenário adotado, este método proporcionou estimativas satisfatórias para o dano em qualquer período de tempo. As estimativas para o dano obtidas por este método não diferiram das obtidas pelo método de Cárcamo Romero, discutido a seguir.

\section{Método do valor de mercado (VM)}

O valor de mercado, permite uma estimativa do dano a áreas florestais, porém possui dois inconvenientes: primeiro, em povoamentos jovens, onde a madeira ainda não possuir um valor comercial, o valor do dano seria zero. Segundo, nos períodos onde a madeira possui valor comercial, o estudo de caso demostrou que o valor do dano encontra-se superestimado em relação aos outros métodos, pois refere-se à receita bruta que seria obtida se todo o estoque de madeira fosse vendido.

Este método poderia proporcionar estimativas corretas se o valor de mercado refletisse o real valor do povoamento no momento do dano e se a perda fosse total.. Entretanto, esta estimativa é complexa pois teria que considerar vários fatores, o que possivelmente acabaria inviabilizando o método.

\section{Método McLean (D1)}

O valor de expectativa da terra (Se), conhecido como método de Faustmann ou valor esperado da terra (VET), representa o valor produtivo da terra, ou seja, indica quanto se pode pagar pelo fator terra caso a mesma fosse utilizada para sempre para a produção florestal.

Para o cenário adotado, o método de McLean proporcionou estimativas consistentes para o dano causado a áreas florestais. Entretanto, 
o método não avalia o valor de outros recursos afetados que, mesmo estando vinculados à floresta, não possuem um valor de mercado. Para a avaliação de danos a áreas reflorestadas em questão, o valor de outros recursos não foi considerado, já que o principal objetivo é a produção de madeira destinada para geração de energia.

\section{Método alternativo de Cárcamo Romero (D2)}

O valor do povoamento antes de ocorrer o dano (VSD) fornece o valor líquido do projeto de investimento em reflorestamento na idade de avaliação, t. Considerando que o dano tenha ocorrido no ano zero, a estimativa proporcionada por este estimador será o próprio valor presente líquido (VPL) do projeto do investimento.

Para o cenário analisado, os valores positivos para o VSD significa que o projeto de investimento é economicamente viável, em condições normais e sem a presença de dano, oferecendo um benefício líquido ao produtor.

O valor do povoamento após a ocorrência do dano (VCD), proporcionou resultados negativos por não haver receitas acumuladas já que o dano se verificou antes da ocorrência da colheita, prevista para os 7 anos.

Da observação do Quadro 1, constatase um VSD $>0$ e um $\mathrm{VCD}<0$, confirmando a hipótese de Cárcamo Romero, de que esta situação é característica quando o projeto é danificado antes de ocorrer o primeiro corte.

Também é possível constatar que o investimento em terra (IT) menor que o valor de expectativa da terra (Se), proporcionou um maior valor para o dano, nas mesmas idades, pelo método de Cárcamo Romero que aquele obtido pelo método de McLean, estando estes resultados em conformidade com Cárcamo Romero (1985). Entretanto, para o investimento em terra igual ao valor de expectativa da terra, à mesma taxa de juros e um ciclo de corte, ambos os métodos proporcionam as mesmas estimativas para o dano.

O método de Cárcamo Romero também apresentou estimativas satisfatórias para o dano embora, essas estimativas não tenham diferido das proporcionadas pelo método do valor produtivo da terra (VPT) comentado anteriormente.

\section{CONCLUSÕES}

Como principais conclusões deste trabalho citam-se:

- Os métodos de avaliação de danos abordados permitem às empresas florestais estimarem, em termos monetários, os danos ao material lenhoso, haja visto que muitos produtos ou benefícios das florestas, além de serem de difícil quantificação, às vezes não possuem um valor de mercado.

- Os métodos de determinação de danos a áreas florestais estudados, a exceção do valor de mercado (VM), proporcionaram estimativas consistentes e pouco diferiram entre si. Assim, a decisão de qual método utilizar dependerá dos objetivos do manejador.

- O método do valor de mercado (VM), da forma como foi definido, apresenta o inconveniente de não proporcionar estimativas para o dano em povoamentos jovens sem valor comercial.

\section{AGRADECIMENTOS}

Ao Conselho Nacional de Desenvolvimento Científico e Tecnológico (CNPq) pelo apoio financeiro e à Universidade Federal de Viçosa pelo fornecimento da estrutura e pessoal.

\section{REFERÊNCIAS BIBLIOGRÁFICAS}

ASSOCIAÇÃO BRASILEIRA DE PRODUTORES DE FLORESTAS PLANTADAS ABRAF. Anuário estatístico da ABRAF: ano base 2006. Brasília: 2007, 80p.

CENTRO DE DESENVOLVIMENTO DO AGRONEGÓCIO - CEDAGRO. Disponível em: <http://www.cedagro.org.br $>$. Acesso em: 01 nov. 2007. 
CIANCILLI, P.L. Incêndios florestais prevenção e combate. São Paulo, Nobel, 1981. 169p.

DAVIS, K.P. Forest management regulation and valuation. New York, McGraw-Hill, 1965. 519p.

EMBRAPA FLORESTAS. Produtos e Serviços - Planilha de custos de eucalipto. Disponível em: $<$ http://www.cnpf.embrapa.br/arquivos/Planilha_Eucalipto.pdf $>$. Acesso em: 05 nov. 2007.
McLEAN, D.L. Appraisal of damage to immature timber. Ottawa, Forest Fire Research Institute, 1970. 12p. (Information Report FF$\mathrm{X}-22)$.

CÁRCAMO ROMERO, J.C. Avaliação econômica de danos a áreas reflorestadas com eucalipto, no estado de Minas Gerais. Viçosa: UFV, 1985. 200p. Dissertação (Mestrado em Ciência Florestal)- Universidade Federal de Viçosa, 1985. 